\title{
Analysis on multi-frequency vortex-induced vibration and mode competition of flexible deep-ocean riser in sheared fluid fields
}

\author{
Yilun $\mathrm{Li}^{\text {a, } 1}$, Shuangxi Guo ${ }^{\text {b,c,1 }}$, Weimin Chen ${ }^{\text {b,d,* }}$ \\ a Sino-French Engineering School, Beihang University, Beijing 100191, China \\ ${ }^{\mathrm{b}}$ Key Laboratory of Mechanics in Fluid Solid Coupling System, Institute of Mechanics, Chinese Academy of Sciences, Beijing 100190, China \\ ${ }^{\mathrm{c}}$ AVIC Composite Corporation LTD, National Key Laboratory of Advanced Composites, China \\ ${ }^{\mathrm{d}}$ School of Engineering Science, University of Chinese Academy of Sciences, Beijing 100049, China
}

\section{A R T I C L E I N F O}

\section{Keywords:}

Vortex-induced vibration

Multi-frequency

Mode competition

Fluid-solid coupling

Long flexible riser

\begin{abstract}
A B S T R A C T
Multi-frequency vortex-induced vibration of flexible riser in lineally sheared fluid fields with different shearing parameters is explored by using the numerical approach. By combining the finite element method with a hydrodynamic model, the approach can consider mode competition based on modal energy and can carry out nonlinearly simultaneously dynamic response in time domain. Our analysis shows that multi-frequency VIV may occur both in non-uniform and uniform fluid fields. And, the behaviors of multi-frequency VIV are different from single-frequency VIV. Because several modes are involved and compete with each other, and consequently the determination of modal excitation region become more complicated. As the towing speed (or the shearing parameter) increases in sheared flow, the average RMS displacement does not regularly rise (or drop), but slightly fluctuates owing to changes of the participating mode and its excitation region. On the other hand, the average RMS stress gradually rises owing to higher-order modes being included. Moreover, it is found that the dominant frequency distributing along structural span significantly changes with the towing speed, and the length of the first dominant frequency gets smaller due to larger shearing parameter along with more intense competition between the participating modes.
\end{abstract}

\section{Introduction}

As the offshore industry moves towards deeper ocean, some floating platforms such as spar, tension leg and semi-submersible platforms have been put into services. Marine risers of these deep-ocean platforms are employed to transport gas and oil or optical and electrical information. The fundamental issues, such as vortex-induced vibration (VIV) and fatigue problems, of these long flexible risers experiencing ocean current (or wave) become more complicated as water depth increases. On the structural side, the dynamic characteristics of slender riser usually presents low-frequency and high-density natural modes due to its large structural flexibility. On the fluid side, the distribution of fluid field, in terms of velocity value and direction, is no longer uniform. Moreover, the shedding mode or frequency of wake vortex may vary, or even be grouped in cells, along structural span due to span-wise coupling of vortices (Mukhopadhyay et al., 1999; Lucor et al., 2001; Williamson and Govardhan, 2008). Therefore, VIV of a long flexible riser experiencing non-uniform flow often presents new phenomena such as multi-frequency (or called as multi-mode) VIV, travelling wave and wide-band random vibrations (Chen et al., 2016; Violette et al., 2010; Sarpkaya, 2004; Facchinetti, 2004; Huera-Huarte and Bearman, 2009). Since natural frequencies of slender riser are dense, i.e. the frequencies of adjacent modes are very close to each other, several modes with different frequencies are excited. Further understanding of multi-frequency VIV in non-uniform flow is significantly challenging.

In recent years, increasing researches about multi-frequency VIV have been reported (Vandiver et al., 1996; Huse et al., 1998; Lie and Kaasen, 2006; Dong and Karniadakis, 2005; Tang et al., 2007; Srinil, 2010; Huera-Huarte et al., 2006; Huang et al., 2011; Jaiswal and Vandiver, 2007; Tognarelli et al., 2004; Marcollo and Hinwood, 2002; Zhang et al., 2013), which were mostly based on experiments. Lie et al. implemented a large-scale tests (Huse et al., 1998; Lie and Kaasen, 2006), he studied if and under which circumstances the riser motions would be single-frequency or multi-frequency. He found that in general the

\footnotetext{
* Corresponding author. Key Laboratory of Mechanics in Fluid Solid Coupling System, Institute of Mechanics, Chinese Academy of Sciences, Beijing 100190, China.

E-mail address: wmchen@imech.ac.cn (W. Chen).

1 These authors contributed equally to this work.
} 
response was irregular (i.e. broad-banded) and that the degree of irregularity increases with the flow speed. In some tests distinct spectral peaks could be seen, corresponding to a dominant mode. Huera-Huarte et al. (2006). gave the force distribution of a riser in a stepped current by using experimental data and the finite element method. The experiments showed a correlation between the mean drag and the transverse response along the cylinder. Huang et al. (2011). used the measured data in uniform current to study the drag amplification and considered the spatial variation of amplitude. By using the semi-empirical wake oscillator model, Srinil (2010) found that multiple modal responses overlap in specific velocity ranges and the lock-in band width is mode-dependent. And, Zhang et al. (2013). found that the unstable zone may grow and a small excitation can induce large dynamic response.

As for the mechanism of multi-frequency VIV and its mode competition, it is still somewhat unclear and has drawn some interesting discussions. Jaiswal et al (Jaiswal and Vandiver, 2007). used the concepts of "time sharing" to describe the "mode switching" along the time coordinate, while Tognarelli et al. (2004). called it "space sharing". Violette et al. (2010). performed a linear stability approach to identify the mode switching of two excited modes in cable VIV. He theoretically explained this behavior based on the linear stability approach, regarding different modes could be excited at different and coincident time instants. Marcollo and Hinwood (2002) examined the area where a cylinder's VIV could vary from single-frequency to multimodal. He found an interesting and unexpected mechanism that the damping region of higher-frequency mode may provide power-in effect to support other modes.

Still, there are some problems remain interesting and, particularly, mode competition and its expression need further studies. In this study, multi-frequency VIV in sheared fluid profiles, i.e. sheared flows with different shearing intensities, is examined and compared with precious single-frequency VIV. A nonlinearly numerical approach, where FEM is combined with the hydrodynamic model and mode competition can be particularly considered based on modal energy, is developed. Then the effects of towing speed (or shearing parameter) on displacement, stress and dominant frequency (or participating mode) distribution are studied, and mode competition during VIV is discussed.

\section{Numerical simulation based on finite element method and the hydrodynamic model}

The governing equation of a slender riser (see Fig. 1), generally regarded as a tensioned Euler beam, undergoing VIV can be written as

$m \frac{\partial^{2} y(z, t)}{\partial t^{2}}+\gamma \frac{\partial y(z, t)}{\partial t}+E I \frac{\partial^{4} y(z, t)}{\partial z^{4}}-T \frac{\partial^{2} y(z, t)}{\partial z^{2}}=f(z, t)$

where $y(z, t)$ is the displacement which is in cross-flow direction (here

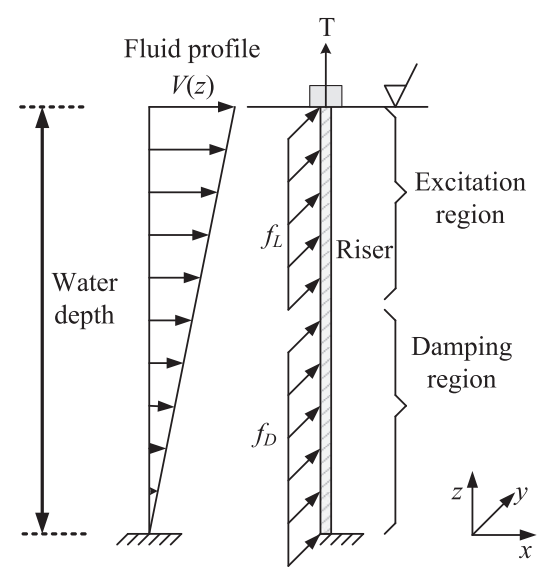

Fig. 1. Scheme of a riser in sheared fluid profile. the coupling of cross-flow and in-line motion is not considered), $m$ and $\gamma$ are the structural mass and damping per unit length; $E I$ and $T$ are the bending stiffness and axial tension. $f(z, t)$ is the hydrodynamic force per unit length consisting of the vortex-induced lift force $f_{L}(z, t)$ and fluid force $f_{D}(z, t)$. Theoretically, to get the solution of Equation (1) is pretty difficult. Because the vortex-induced lift force $f_{L}(z, t)$ actually couples with body motion and can hardly have a precisely theoretical expression owing to complicated wake field during lock-in. And, by now, CFD of VIV still needs expensive cost of computation resource, especially for a deep water riser at high Reynolds number.

Here, a numerical approach based on finite element method and the hydrodynamic model is introduced to analyze the nonlinear simultaneously dynamic response of a riser undergoing VIV. This approach can capture the main VIV's traits during lock-in under an acceptable computation cost by avoiding CFD. More specifically, the riser is divided into finite beam elements upon which the hydrodynamic models are loaded. Then direct numerical integration is used to solve the governing equation and get dynamic response in time domain.

\subsection{Structural finite element model}

The slender riser is assumed as an Euler beam and can be uniformly divided into finite elements. Here, two-node Euler beam element (Craig, 1981 ) is used and the number of beam elements is $N$. For representativeness and simplicity, only one translation displacement $y_{i}$, $i=1,2, \ldots, N+1,(N+1$ is the total number of nodes), and one rotation $\theta_{i}, i=1,2, \ldots, N+1$ per node, are considered. The displacement function of the beam element is written as

$y(\xi)=\sum_{i=1}^{2} \varphi_{i}^{0}(\xi) y_{i}+\sum_{i=1}^{2} \varphi_{i}^{1}(\xi) \theta_{i}$

where $\quad \varphi_{1}^{0}(\xi)=1-3 \xi^{2}+2 \xi^{3}, \quad \varphi_{2}^{0}(\xi)=3 \xi^{2}-2 \xi^{3} \quad$ and $\varphi_{1}^{1}(\xi)=\left(\xi-2 \xi^{2}+\xi^{3}\right) / l_{e}, \varphi_{2}^{1}(\xi)=\left(\xi^{3}-\xi^{2}\right) / l_{e} . \xi$ is the internal coordinate of beam element, $\xi=\left(z-z_{1}\right) / L, \quad 0 \leq \xi \leq 1 . l_{e}$ and $L$ are the element length and overall structural length respectively. The element matrices are respectively as mass matrix $\mathbf{M}^{e}=\int_{l_{e}} \rho \boldsymbol{\varphi}^{T} \boldsymbol{\varphi} d \xi$, stiffness matrix $\mathbf{K}^{e}=\int_{l_{e}} \mathbf{B}^{T} K^{\prime} \mathbf{B} d \xi$ and geometry stiffness matrix $\mathbf{K}_{t}^{e}=\int_{l_{e}} \overline{\mathbf{B}}^{T} \overline{\mathbf{B}} d \xi$, where $\boldsymbol{\varphi}=\left[\begin{array}{llll}\varphi_{1}^{0} & \varphi_{1}^{1} & \varphi_{2}^{0} & \varphi_{2}^{1}\end{array}\right]$ is the element deformation function. Coefficient matrices $\mathbf{B}=\frac{d^{2} \boldsymbol{\varphi}}{d x^{2}}, \overline{\mathbf{B}}=\frac{d \boldsymbol{\varphi}}{d x}$ and $K^{\prime}=E I$.

Then the governing equation of the riser with many degrees of freedom can be written as:

$\mathbf{M Y}+\mathbf{C} \dot{\mathbf{Y}}+K Y=\mathbf{F}$

where $\mathbf{M}, \mathbf{C}$ and $\mathbf{K}$ are the structural mass, damping and stiffness matrices respectively, which can be assembled by the corresponding element matrices. $\mathbf{Y}$ and $\mathbf{F}$ are the displacement and load vector of the nodes.

For case of small structural damping, the Rayleigh damping can be used and is written as

$\mathbf{C}=a \mathbf{M}+b \mathbf{K}$

where $a$ and $b$ are positive constants of which values can be determined by experiments, or approximately, the natural frequencies of structure as follows:

$a=\frac{2 \omega_{1} \omega_{2}\left(\zeta_{1} \omega_{2}-\zeta_{2} \omega_{1}\right)}{\omega_{2}^{2}-\omega_{1}^{2}}, \quad b=\frac{2\left(\zeta_{2} \omega_{2}-\zeta_{1} \omega_{1}\right)}{\omega_{2}^{2}-\omega_{1}^{2}}$

where $\zeta_{j}$, and $\omega_{j}(j=1,2)$ are respectively damping ratio and natural frequency of mode $j$. Generally, the value of structural modal damping ratio is 3 percent, or $\zeta_{1}=\zeta_{2}=0.03$ (Craig, 1981; Vandiver and Li, 1999).

The vortex-induced lift force $f_{L}(z, t)$ and the drag force $f_{D}(z, t)$ exerted by the ambient fluid are applied at the nodes respectively in the excita- 
tion and damping region along the structural length. The element load in terms of element node is $\mathbf{F}^{e}=\int_{l_{e}} q \boldsymbol{\varphi}^{T} d \xi$. Element mass are equally divided at two nodes of each beam element.

Finally, the displacement is solved at discrete times with a fixed integration times step $\Delta t\left(\Delta t \leq(1 / 50) T_{\min }\right), T_{\min }$ is the minimum natural period of all participating modes. By using a Newmark-Beta representation for the velocity and the displacement at discrete times

$\left\{\dot{y}_{t+\Delta t}\right\}=\left\{\dot{y}_{t}\right\}+\left[(1-\delta)\left\{\ddot{y}_{t}\right\}+\delta\left\{\ddot{y}_{t+\Delta t}\right\}\right] \cdot \Delta t$

$\left\{y_{t+\Delta t}\right\}=\left\{y_{t}\right\}+\left\{\dot{y}_{t}\right\} \cdot \Delta t+\left[\left(\frac{1}{2}-\alpha\right)\left\{\ddot{y}_{t}\right\}+\alpha\left\{\ddot{y}_{t+\Delta t}\right\}\right] \cdot \Delta t^{2}$

the dynamic equation can be solved. The initial condition is that both the initial displacement and velocity are zero. The boundary conditions at the two pinned ends of the riser are:

$$
\begin{aligned}
& x(0, t)=0 \\
& \partial^{2} x(0, t) / \partial z^{2}=0
\end{aligned} \text { and } \quad \begin{aligned}
& x(L, t)=0 \\
& \partial^{2} x(L, t) / \partial z^{2}=0
\end{aligned}
$$

\subsection{Hydrodynamic model and its excitation region based on mode competition mechanism}

\subsubsection{Hydrodynamic model}

Two hydrodynamic expressions are used to respectively model the fluid force $f_{D}(z, t)$ and vortex-induced lift force $f_{L}(z, t)$. Here a basic assumption is that the hydrodynamic force depends on the body motion so as to taken into account of the coupling between structural and fluid dynamics. Firstly, given the fact that the riser is a slender cylinder body, the fluid force $f_{D}(z, t)$ can be expressed by virtue of the Morison's formula, i.e.

$f_{D}(z, t)=(1 / 2) C_{D} \rho D \dot{y}(z, t)|\dot{y}(z, t)|+(1 / 4) \pi C_{A} \rho D^{2} \ddot{y}(z, t)$

where $\rho$ is the density of the fluid, and $D$ is the diameter of the riser. $C_{D}$ and $C_{A}$ are the drag and added mass coefficients respectively, of which the values are $C_{A}=1.0$ and $C_{D}=1.1$ for a flexible riser with large aspect ratio (Sarpkaya, 2004; Vandiver and Li, 1999).

Secondly, the expression of vortex-induced lift force $f_{L}(z, t)$ is more complicated because VIV has always been a challenging issue concerning the interaction between fluid and structural dynamics. Different from the originally constant lift coefficient $C_{L}$, i.e. $f_{L}=(1 / 2) \rho V^{2} C_{L} D$, new approaches of lift force were proposed (Chen et al., 2016; Sarpkaya, 2004; Vandiver and Li, 1999), which depend on body motion during lock-in. Because the coupling between structural and fluid dynamics were taken into accounts, these new approaches are more accurate and reasonable. Sarpkaya (2004) pointed out that for slender riser with higher Reynolds numbers (e.g. ranging among $\left.(0.3 \sim 2.0) \times 10^{6}\right)$ the nonlinear expression with respect to structural motion is more reasonable. Here, the third-order polynomial of the structure velocity originally suggested by Chen (Chen et al., 2016) is used to model the lift coefficient as follow

$\left.f_{L}(z, t)=p_{f} C_{L 0} \sin (\omega t)+p_{f} C_{1} \dot{y}(z, t)+p_{f} C_{2} \dot{y}^{2}(z, t)+p_{f} C_{3} \dot{y}^{3}(z, t)\right)$

where $p_{f}=(1 / 2) \rho D V^{2}$. The values of coefficients $C_{L 0}, C_{1}, C_{2}$ and $C_{3}$ can be derived by fitting experimental or CFD data (Lucor et al., 2001; Williamson and Govardhan, 2008; Sarpkaya, 2004; Vandiver et al., 1996; Huse et al., 1998; Lie and Kaasen, 2006; Huera-Huarte et al., 2006; Huang et al., 2011). Chen et al. (2012) gave an approach to calculate the coefficients by fitting experimental data.

The main reason equation (8) is used is that it is able to capture, to some extents, features of VIV. For examples, the feature of self-excitation is represented by the first term $p_{f} C_{L 0} \sin (\omega t)$ together with the second term $p_{f} C_{1} \dot{y}(z, t)$, which increases as response increases $\left(C_{1}\right.$ is required to be positive); the feature of self-limitation, i.e. structural response begins to drop when its value reaches to a certain number $\left(\bar{y}_{\max }=1.5\right.$ or 2.0$)$, is represented by the nonlinear terms with higher orders of structural motion in Eq. (7), $\rho_{f} C_{2} \dot{y}^{2}(z, t)$ or $\rho_{f} C_{3} \dot{y}^{3}(z, t)$ (at least one of the coefficients $C_{2}$ and $C_{3}$ is negative). And, this model automatically captures the span coherence behavior of a flexible riser's VIV in some way because the lift force is non-uniform owing to the axially varying amplitude of riser's response.

\subsubsection{Excitation region based on mode competition and its expression}

For case of multi-frequency VIV, to determine the excitation region, upon which vortex-induced lift force is acted, becomes complicated. Because overlap between adjacent excitation regions may occur if several modes simultaneously participate in the response. Here, to eliminate this overlap, modal competition is particularly introduced to measure excitation region so that the mode more powerful would be more likely to participate into the response, or even, finally would become the dominant mode.

As we know, for case of single-frequency VIV, the modal excitation location can be simply determined according to modal reduced velocity $V_{r n}(\mathrm{z}, n)=V(z) / D f_{n}$, i.e. $4 \leq V_{r n}(z) \leq 12$. However, if the fluid profile is sheared, the distribution of reduced velocity along riser length is nonuniform. Or for case of multi-frequency vibration, at a certain location $z$, there may exist multiple participating modes of which the modal reduced velocities all satisfy $4 \leq V_{r n}(z) \leq 12$.

Here, it is proposed that there are competitions between all potentially participating modes, and the modal weight based on its modal energy is used to determine excitation region. Analyzing the modal data of VIV tests (Lie and Kaasen, 2006; Chaplin et al., 2005), we found that the participating modes varied from mode 20 to 10 as the reduced velocity changes from 4 to 12 . Moreover, appearance of a new excitation mode is usually accompanied by disappearance of previously existing modes. Or, the new excitation mode with increasingly large power could overcome the previous one with decreasing power until it disappears in overall dynamic response (see Figs. 9, 15 and 16 there in the paper by Chaplin et al. (2005).). And, based on the large-scale tests in shear flow (Huse et al., 1998; Lie and Kaasen, 2006), we noted that the modal weights of all participating modes distribute unequally and, often, there are a few modes (e.g. around 1-3 modes) mainly dominating the vibration response (or with higher values of modal weight).

So the modal weight based on modal energy (Chen et al., 2016) can be written as:

$P_{n}=\left[\int_{L_{n}}(1 / 2) C_{L} \rho D V^{2}(z) \varphi_{n}^{2}(z) d z\right]^{2} / \int_{L-L_{n}} r_{h}(z) \varphi_{n}^{2}(z) d z+\int_{L} r_{s}(z) \varphi_{n}^{2}(z) d z$

where $r_{h}(\boldsymbol{z})$ and $r_{s}(\boldsymbol{z})$ are the hydrodynamic and structural damping respectively. $L_{n}$ is the modal excitation length according to the reduced velocity. $\phi_{n}(z)$ is the modal shape. Since the location of excitation force is of important to the modal excitation energy, i.e. the excitation force applied at anti-node of this mode would induce a much larger response than the force at the node. Thus, modal shape $\phi_{n}(z)$, representing the location of force, is involved in Eq. (9). Then the original length of excitation region for participating modes shrinks, in a way of inversely proportional to its modal energy, till the overlap disappears, i.e. $L_{n} / L_{n+1}=P_{n} / P_{n+1}$.

Finally, to select the modes that are most likely to participate into the dynamic response, only the first six modes which have the largest values of modal energy are considered; or, if the modal energy is 85 percent lower than the first mode, the mode will be neglected. 


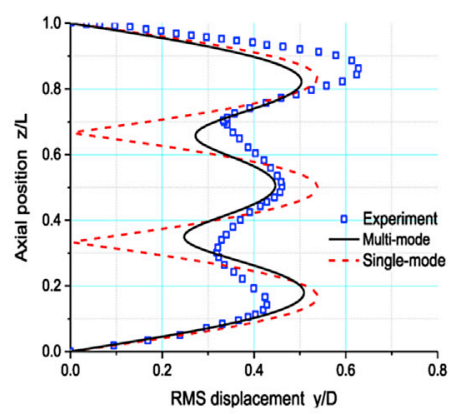

(a) The RMS displacements of respectively single-frequency and multi-frequency VIV
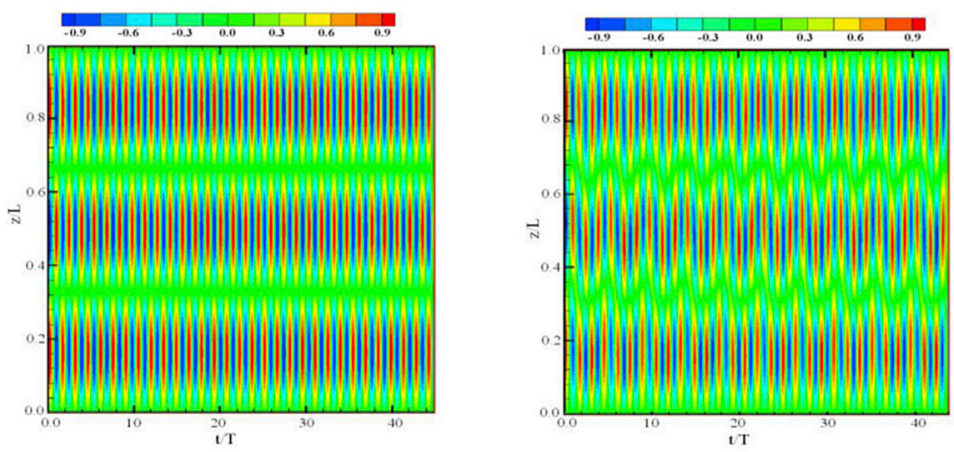

(b) The temporal-spatial evolution of displacement of respectively single-frequency (left) and

\section{multi-frequency (right) VIV}

Fig. 2. Multi-frequency and single-frequency VIV of a flexible cable in uniform fluid profile, based on our numerical simulations against the experimental results.

\section{Multi-frequency VIV responses and its discussions}

\subsection{Comparison of single-frequency and multi-frequency VIV against experiments in different fluid profiles}

The numerical results of multi-frequency and single-frequency VIV are compared with the experimental results, i.e. a flexible cable in uniform flow by Trim (Trim et al., 2005) and a flexible riser in non-uniform flow by Huse (Huse et al., 1998) and Lie (Lie and Kaasen, 2006).

\subsubsection{The flexible cable in uniform flow}

The cable length is $38.00 \mathrm{~m}$, and the outer/inner diameter is $0.027 \mathrm{~m} /$ $0.024 \mathrm{~m}$. The structural mass per unit length is $0.76 \mathrm{~kg} / \mathrm{m}$, and the bending stiffness is $37.20 \mathrm{Nm}^{2}$. Two dynamic responses, i.e. the singlefrequency VIV involving only the mode 3 and the multi-frequency VIV involving the modes 3 and 4, are carried out. The RMS displacement and the temporal-spatial evolution are shown in Fig. $2 \mathrm{a}$ and $\mathrm{b}$ respectively. Generally speaking, both results give satisfied agreements with experiments in term of the dominant mode and the displacement phase. For examples, both numerical RMS curves show mode 3 is the dominant mode, which is consistent with the experiment; and the numerical offset of displacement peak (or trough) location are acceptable, around 7-10\%.

If considering the displacement values, the multi-frequency VIV agrees with the experiment better than the single-frequency. For examples, the span-averaged RMS displacements (non-dimensionalized as $y / D$ ) are respectively 0.36 (multi-frequency) and 0.34 (single-frequency) compared with 0.39 of the experiment; the averaged-value of the 3 peaks are respectively 0.48 (multi-frequency) and 0.54 (single-frequency) compared with 0.50 of the experiment. Moreover, the average values of the two troughs of single-frequency is just 0.0068 that is much lower than the experimental value 0.32 while the multi-frequency value is 0.26 . That is to say, the displacement of single-frequency VIV just agree with the experiment at the peaks, but remarkably diverges from the experiment near the two troughs. The temporal-spatial evolutions (see Fig. 2b) shows the slight travelling wave, observed in the experiment, is only seen in the multi-frequency VIV, while the single-frequency VIV is characterized as a standing wave.

\subsubsection{The flexible riser in non-uniform flow}

The riser in a large-scale field experiment (Huse et al., 1998; Lie and Kaasen, 2006), of which the aspect ratio is quite large (3000), is taken as the example. Its length is $90 \mathrm{~m}$, and its outer and inner diameters are respectively $0.030 \mathrm{~m}$ and $0.026 \mathrm{~m}$. The Young's modulus of the riser's material is $210 \mathrm{GPa}$, and the top tension of the riser is $3700 \mathrm{~N}$. The dynamic characteristics of the riser are shown in Fig. 3 which shows the natural frequencies and the potential modes, e.g. the modes 10 to 29 according $0.40 \leq V_{s t} \leq 1.20$. The VIV responses at ten towing speeds

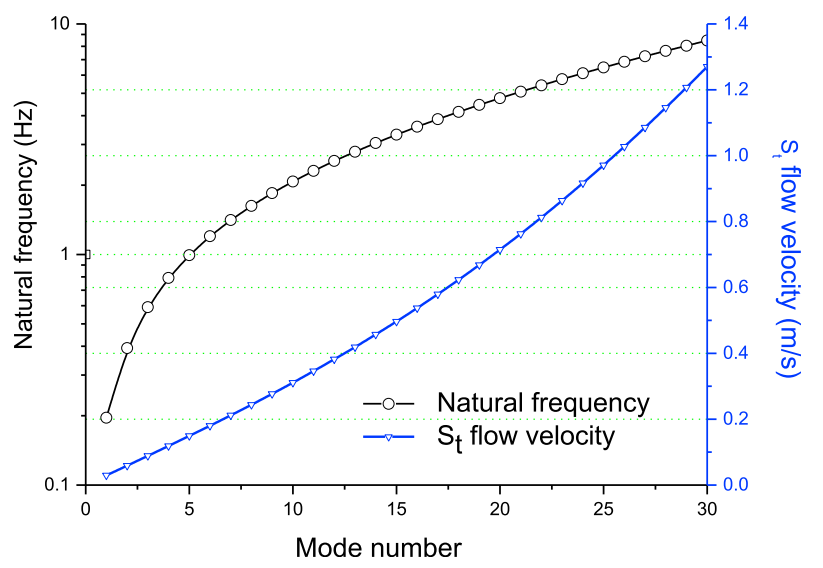

Fig. 3. The frequencies and the corresponding Strouhal flow velocity of the natural modes of the riser, and the Strouhal velocity is $V_{s t}=5 f_{n} D$. 


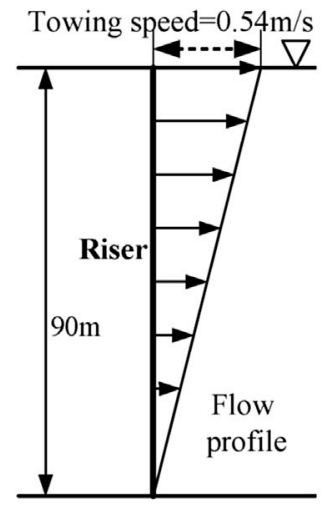

(a) The fluid profile

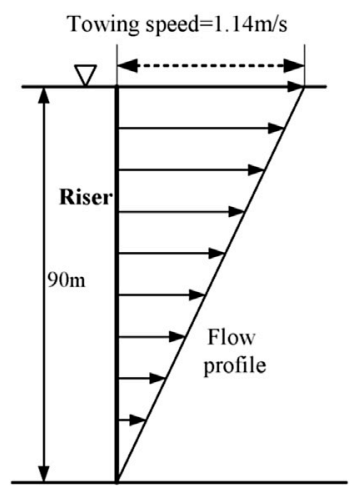

(d) The fluid profile
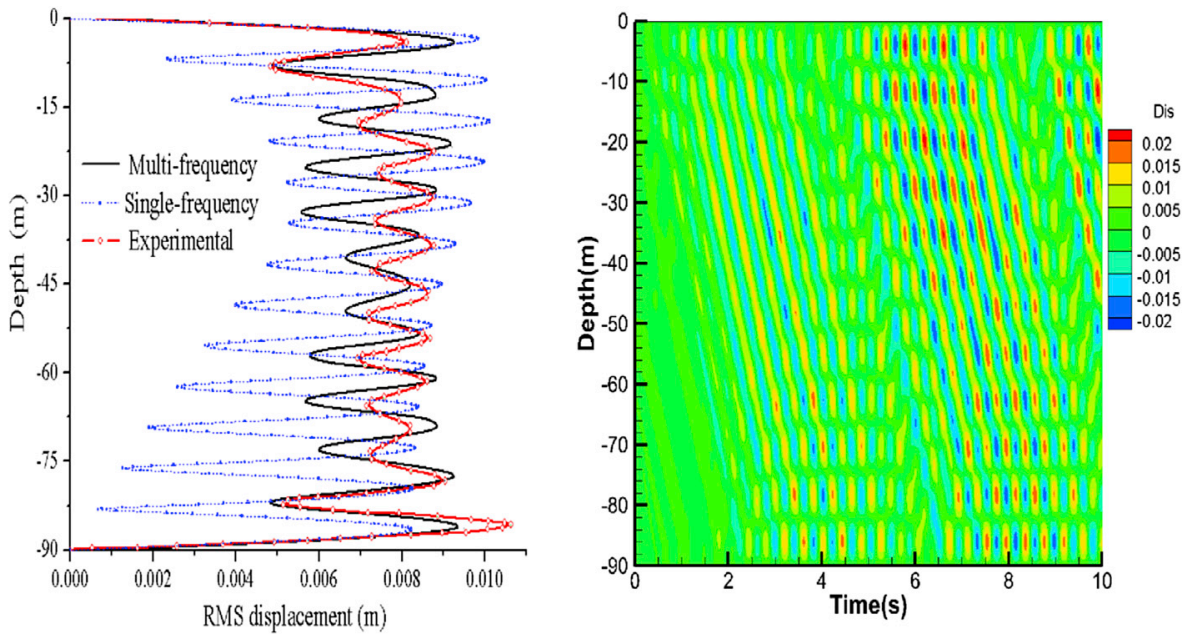

(b) The RMS displacement

(c) The temporal-spatial evolution of along riser span

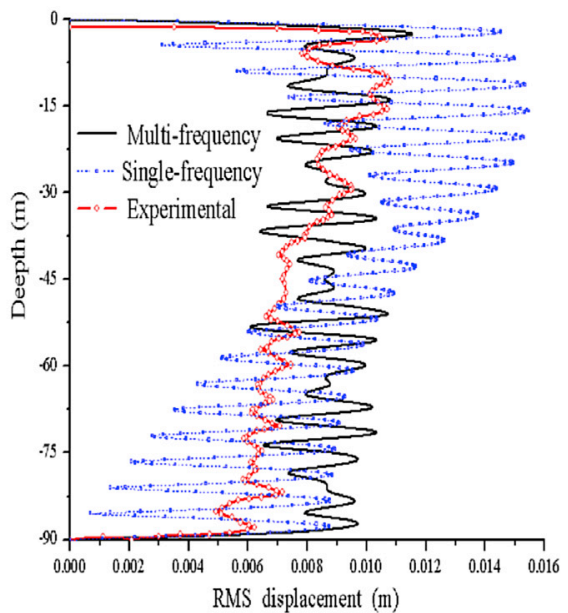

the displacement

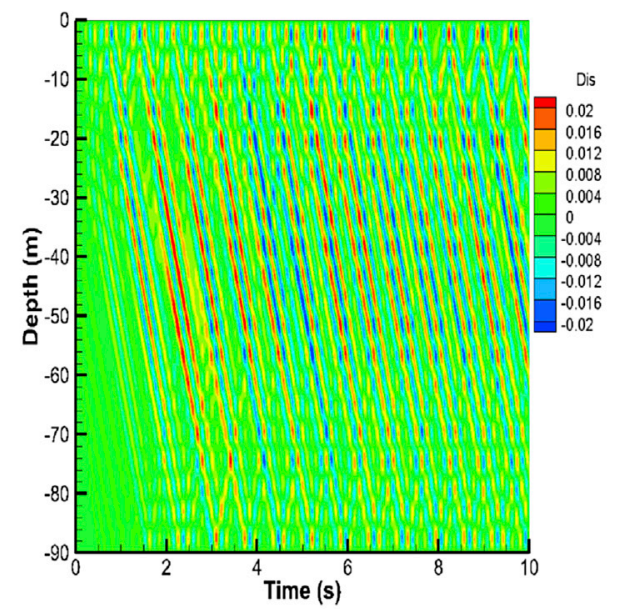

(f) The temporal-spatial evolution of the displacement

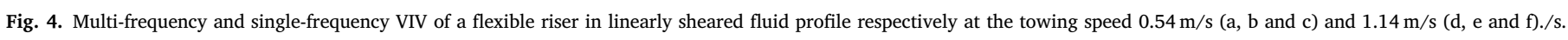

were simulated, and the selected results, at two typical towing speeds of $0.54 \mathrm{~m} / \mathrm{s}$ and $1.14 \mathrm{~m} / \mathrm{s}$, are presented.

The RMS displacement is plotted against the experiments in respectively Fig. $4 \mathrm{~b}$ (at towing speed $0.54 \mathrm{~m} / \mathrm{s}$ ) and Fig. 4e (at towing speed $1.14 \mathrm{~m} / \mathrm{s}$ ). As a comparison, the single-frequency VIV of the dominant mode is plotted too. Table 1 shows some displacement values of the two numerical simulations against the experiments.
Similarly with the case of uniform flow, it is seen that the displacement of the multi-frequency VIV agree with the experiments much better than the single-frequency VIV. Taking the speed of $0.54 \mathrm{~m} / \mathrm{s}$ as an example (see Table 1), the span-averaged RMS displacements are respectively $0.0073 \mathrm{~m}$ (multi-frequency) and $0.0065 \mathrm{~m}$ (single-frequency) compared to $0.0075 \mathrm{~m}$ of the experiment. It is noted that the differences of the trough-averaged values between the numerical and the experiment

Table 1

RMS displacement comparisons between the numerical results and the experiments (Displacement unit: $\mathrm{m}$ ).

\begin{tabular}{|c|c|c|c|c|c|c|}
\hline Towing speed & Model & Span-average displacement & Peak-average displacement & Trough -average dis. & Average-error of peak offsets & Average-error of trough offsets \\
\hline \multirow[t]{3}{*}{$0.54 \mathrm{~m} / \mathrm{s}$} & Exp. & 0.0075 & 0.0088 & 0.0068 & & \\
\hline & M-F VIV & 0.0073 & 0.0090 & 0.0055 & $7.01 \%$ & $11.40 \%$ \\
\hline & S-F VIV & 0.0065 & 0.0091 & 0.0033 & unmatching & unmatching \\
\hline \multirow[t]{3}{*}{$1.14 \mathrm{~m} / \mathrm{s}$} & Exp. & 0.0078 & 0.0081 & 0.0071 & & \\
\hline & M-F VIV & 0.0080 & 0.0092 & 0.0072 & $10.19 \%$ & $14.54 \%$ \\
\hline & S-F VIV & 0.0092 & 0.0118 & 0.0062 & $17.20 \%$ & $21.88 \%$ \\
\hline
\end{tabular}

Notes: 1) "M-F VIV" and "S-F VIV" mean Multi-Frequency VIV and Single-Frequency VIV respectively.

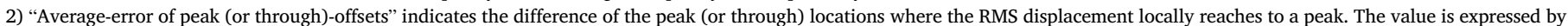
relative error.

3) "Anmatching" means the dominant mode of the single-frequency VIV does not match with the experiment. 
ones are larger, i.e. $19.1 \%$ and $51.4 \%$ respectively. The reason might be there were more and random modes participating into the response during experiments but selected modes are involved in our numerical simulation.

Considering then the dominating mode and phase, the dominating mode of multi-frequency VIV is consistent with the experiment while the single-frequency VIV is a different from the experiment. For example, at towing speed $0.54 \mathrm{~m} / \mathrm{s}$, the dominating modes are mode 13 , mode 11 and mode 11 for the single-, multi-frequency VIV and experiment respectively. If comparing the phase, the offset of peak (or trough) of multifrequency are acceptable, e.g. $7.01 \%$ (or $11.40 \%$ ).

Considering last the wave type. The evolution of displacement versus time and riser span, see Fig. $4 \mathrm{c}$ and $\mathrm{f}$, show the response is a mix of standing wave and travelling wave. That is also reported in References (Srinil, 2010; Bourguet et al., 2013). Particularly, standing wave happens at the top/bottom end. Because at the two ends, it is easier for the propagating wave to meet the one reflecting from the end and then to form a standing wave before it declines to a small value or zero. Additionally, the travelling wave becomes more obvious at higher towing speed $(1.14 \mathrm{~m} \mathrm{~s})$. In this case, higher-order modes are excited, and the damping effect is faster for these modes with higher modal order. Thus the vibration excited at higher frequency near the top-end may decline rapidly into a pretty little, even zero, value before it meet the reflecting wave to form a standing wave.

\subsection{Influences of towing speed on the displacement and stress}

The time histories and temporal power spectral density (PSD) of displacements at two typical points, which have the maximum and minimum RMS displacement, are plotted in Fig. 5 and Fig. 6 for towing speed 0.54 and 1.14 respectively. Figs. 5 and 6 show both the frequency and band width change with the towing speed: as the speed increases

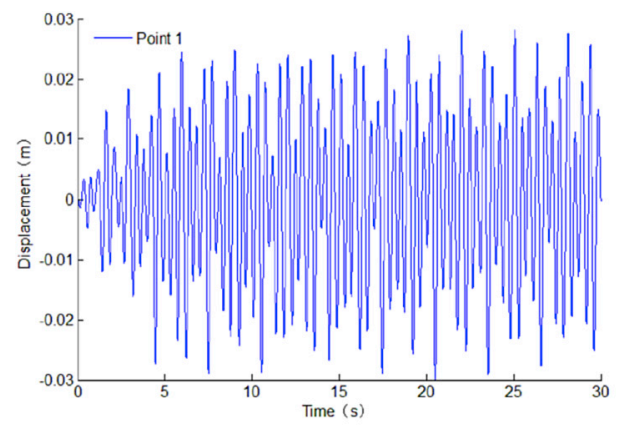

(a) Time history of the maximum displacement

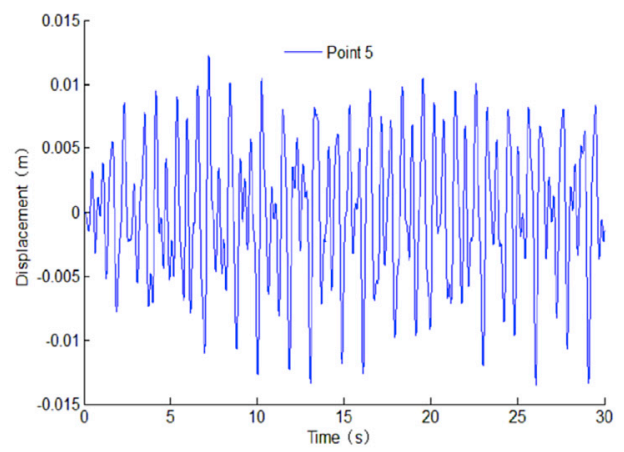

(c)Time history of the minimum displacement from 0.54 to $1.14 \mathrm{~m} / \mathrm{s}$, the highest modal order increases from 16 to 24 , while the band width of excited frequencies broadens, i.e. from $0.99 \mathrm{~Hz}-3.29 \mathrm{~Hz}$ (mode $5 \sim$ mode 16 ) to $0.50 \mathrm{~Hz}-6.29 \mathrm{~Hz}$ (mode $2 \sim$ mode 24).

Interestingly, involvement of more and higher-order modes does not necessarily induce larger displacement, see Fig. 7a. The span-averaged RMS displacement is just around $0.0078 \mathrm{~m}$, e.g. $0.0073 \mathrm{~m}$ for speed $0.54 \mathrm{~m} / \mathrm{s}$ compared to $0.0080 \mathrm{~m}$ for speed $1.14 \mathrm{~m} / \mathrm{s}$. As towing speed increases, different modes participate into (or drop out of) dynamic response while the excitation region for a certain mode change too. Thus, it is uncertain that whether the overall displacement would get larger or smaller. Or, the displacement just slightly change rather than regularly rise (or drop).

However, as the towing speed increases, the stress obviously rises, see Fig. 7b. Because new modes with higher-order number are excited, of which the modal curvatures, or the modal stress get larger. It should be noted that the higher level of stress with higher frequency may impact the fatigue performance of structure, this is really a serious concern of riser safety in ocean engineering.

\subsection{Distribution of dominating frequencies along riser span}

The distributions of dominant frequencies along riser length are presented in Fig. 8. Several peak frequencies can be identified according to the peaks of displacement power spectrum (the spectrum-figures are not presented here in this paper, and the example plots of displacement spectrum can be seen in Fig. $5 \mathrm{~b}$ and d) at different points along riser span. Among these peak frequencies, the first two frequencies which have the largest two values of dominant length along riser span are named as the first-dominant and second-dominant frequency respectively. The rest frequencies are named as other-dominant frequency.

Comparing the results of the two speeds (Fig. 8a and b), it is seen that

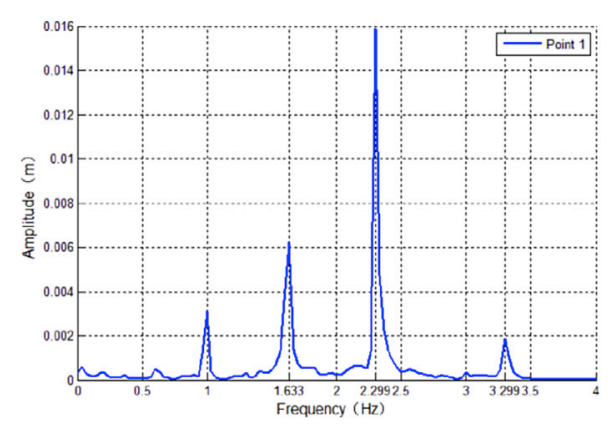

(b) Frequency spectrum of the maximum displacement

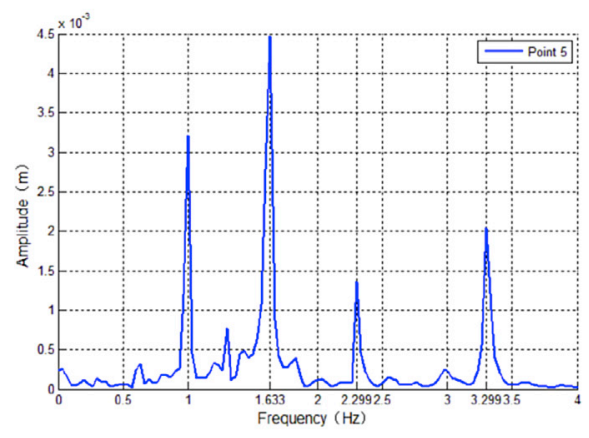

(d) frequency spectrum of the minimum displacement

Fig. 5. Time history of displacement and frequency spectrum of some points along the riser span, at towing speed $0.54 \mathrm{~m} / \mathrm{s}$. 
span, at towing speed $0.54 \mathrm{~m} / \mathrm{s}$.

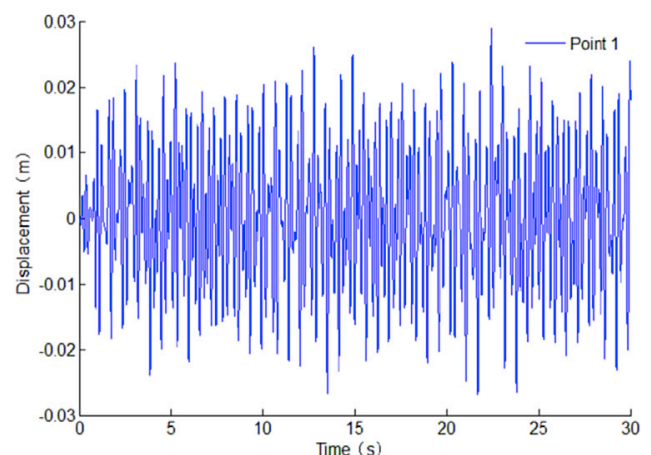

(a) Time history of the maximum displacement

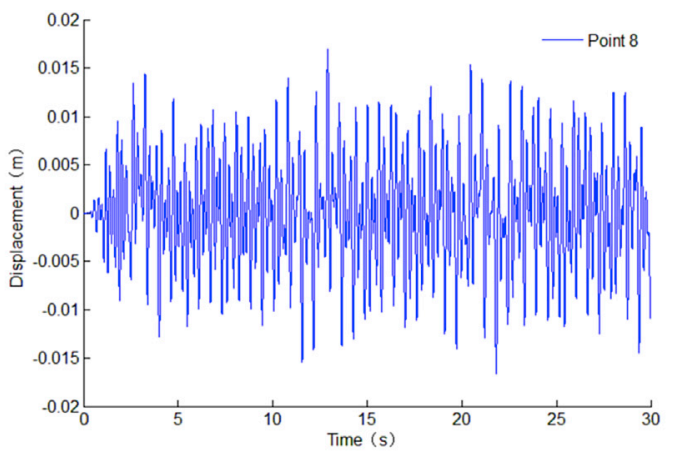

(c)Time history of the minimum displacement

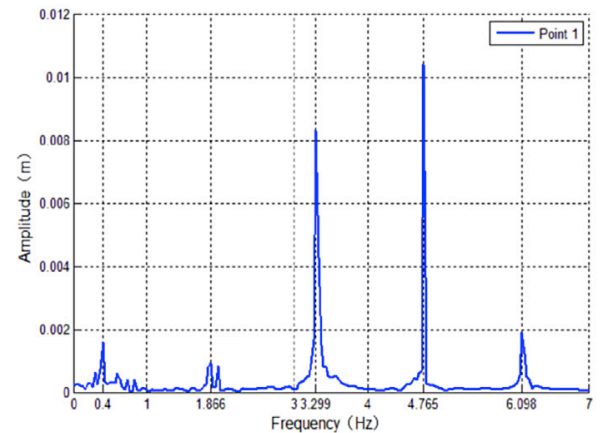

(b) frequency spectrum of the maximum displacement

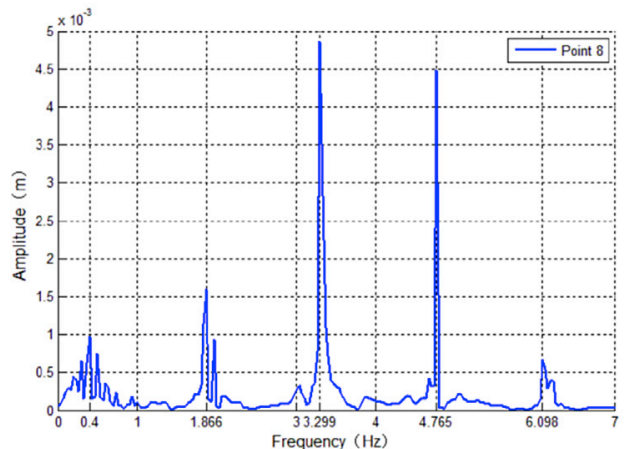

(d) Frequency spectrum of the minimum displacement

Fig. 6. Time history of displacement and frequency spectrum of some points along the riser span, at towing speed $1.14 \mathrm{~m} / \mathrm{s}$.

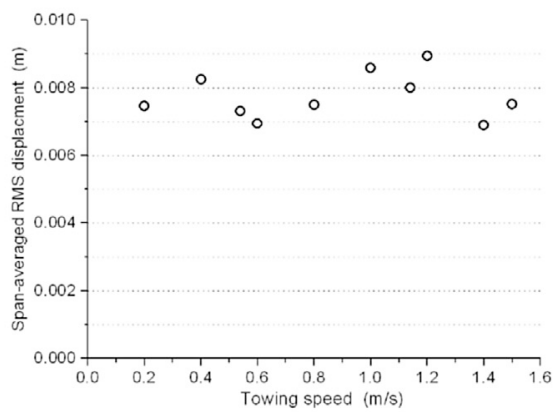

(a) Average RMS displacement versus towing speed

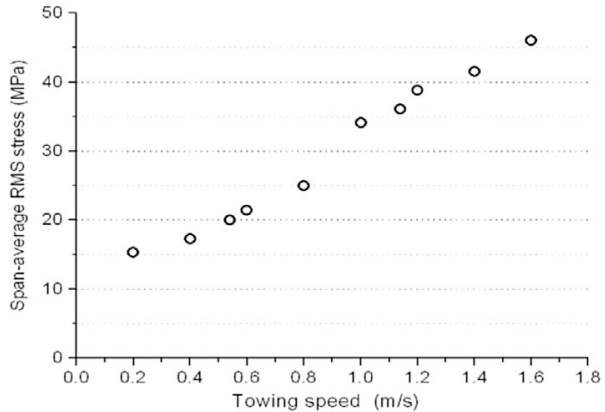

(b) average RMS stress versus towing speed

Fig. 7. Effects of towing speed on vortex-induced vibration response.

the distributions of dominant frequencies change in terms of the band width and length. For case of lower speed $(0.54 \mathrm{~m} / \mathrm{s}$, Fig. $8 \mathrm{a})$, the firstdominant frequency $(2.30 \mathrm{~Hz}$ of mode 11$)$ ranges from $0.5 \mathrm{~m}$ to $89.0 \mathrm{~m}$ depth. While the second-dominant frequency $(1.63 \mathrm{~Hz}$ of mode 8$)$ just scatters at some points among the first-dominant region, as well the other-dominant frequencies. This phenomena was also reported in other examples by Lucor et al. (2001). and Bourguet et al. (2013). They pointed out that it is because of the shedding cells of vertex which have different shapes and frequencies in the wake filed behind the riser undergoing VIV. Here, from our view of structural dynamics, it might because of the multiple modes that simultaneously participate into the response and their nonlinear interactions between each other.
For case of higher speed ( $1.14 \mathrm{~m} / \mathrm{s}$, Fig. $8 \mathrm{~b})$, the band width between the first two dominant frequencies gets wider, e.g. (4.76-3.31) Hz/mode (20-15), compared to $(2.30-1.63) \mathrm{Hz} /$ mode $(11-8)$ at $0.54 \mathrm{~m} / \mathrm{s}$. What's more, the first-dominant region, $0.5 \mathrm{~m}-78.5 \mathrm{~m}$, gets smaller but the second and other dominant frequencies share more region. It is partly because there are more participating modes, ranging from modes 2-26 compared to the modes $3-15$ of $0.54 \mathrm{~m} / \mathrm{s}$ speed, into the dynamic response, as the towing speed gets larger (or the shear intensity, $\left(V_{\max }-V_{\min }\right) / L$ gets larger). In that case, the modal competition gets more intense, and it's harder for a dominant mode to occupy most riser span or it have to share the dominant region with others in some extent. 


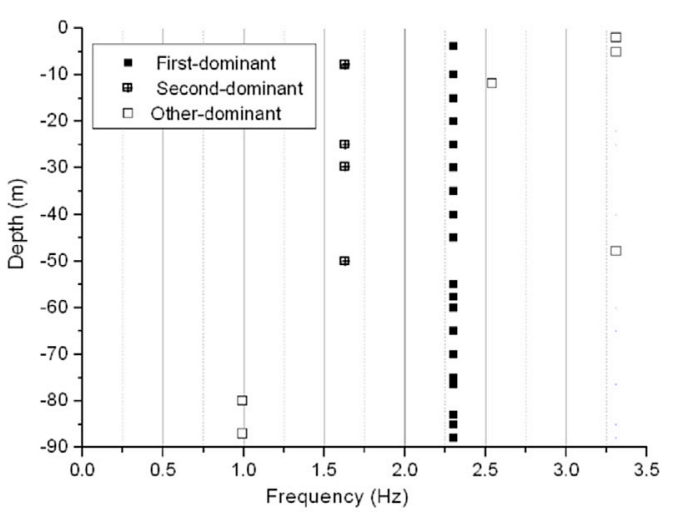

(a) At towing speed $0.54 \mathrm{~m} / \mathrm{s}$

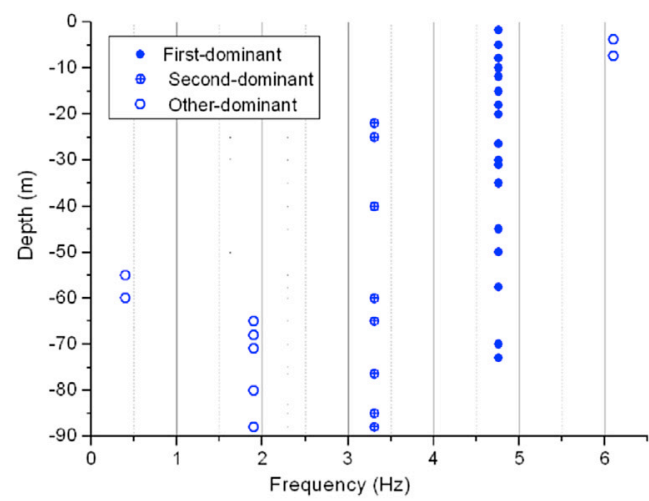

(b) At towing speed $1.14 \mathrm{~m} / \mathrm{s}$

Fig. 8. Distributions of dominant frequencies along the riser span.

\section{Conclusions}

Multi-frequency VIV of long flexible riser in shear fluid profile is explored, which happens not only in non-uniform flow but also in uniform flow, based on the numerical simulations. Particularly, by combining the FEM with the hydrodynamic model, the approach can consider mode competition based on modal energy and carry out nonlinearly simultaneously dynamic response in time domain. The numerical results show that the behaviors of multi-frequency VIV are somewhat different from multi-frequency VIV. As the flow velocity increases, the remarks are as follows:

1) The average RMS displacement does not regularly rise (or like the single-frequency VIV does), but just slightly fluctuates instead. Because different modes participate into (or drop out of) the dynamic response, and the modal excitation region significantly changes along riser span.

2) The RMS stress progressively increases, i.e. linearly increase with the flow velocity. It is principally because more and higher-order modes are excited. The larger value of stress with higher frequency could be a concern of structural strength because of fatigue problem.

3) Interestingly, the distributions of dominant frequencies changes. The length of the first dominant frequency gets smaller due to larger shear parameter along with more intense competition between the participating modes.

Finally, we would like to say that better approach and deeper insight into multi-frequency VIV are still needed. VIV is a nonlinearly coupling of structural and fluid dynamics. It does present some interesting and, even still, not completely understood phenomena such as self-limited, hysteresis and jump/switch between different modes. Fortunately, increasing progresses have been seen on purely numerical simulations such as CFD in recent years, which present more details of hydrodynamic force and body displacement (Williamson and Govardhan, 2008; Sarpkaya, 2004; Bourguet et al., 2013; Lucor and Karniadakis, 2004). Hopefully, these progresses would advance future work on more reasonable approach to make a better prediction of VIV under less computational cost, particularly, for industry design of risers in deep water.

\section{Acknowledgment}

This work was financially supported by the National Natural Science Foundation of China (Grant No. 11232012 and 11372320).

\section{Nomenclature}

$\begin{array}{ll}y(z, t) & \text { displacement in cross-flow direction } \\ m & \text { structural mass per unit length } \\ \gamma & \text { damping per unit length } \\ E I & \text { bending stiffness } \\ T & \text { axial tension } \\ f(z, t) & \text { hydrodynamic force per unit length } \\ f_{L}(z, t) & \text { Vortex-induced lift force } \\ f_{D}(z, t) & \text { fluid force } \\ N & \text { number of beam elements } \\ y_{i} & \text { translation displacement } \\ \theta_{i} & \text { degree of rotation freedom } \\ \xi & \text { internal coordinate of beam element } \\ l_{e} & \text { length of beam element } \\ L & \text { overall riser length } \\ \mathbf{M}^{e} & \text { element mass matrix } \\ \mathbf{K}^{e} & \text { element stiffness matrix } \\ \mathbf{K}_{t}^{e} & \text { element geometry stiffness matrix } \\ \boldsymbol{\varphi} & \text { element deformation function }\end{array}$


M structural mass matrices

C structural damping matrices

K structural stiffness matrices

Y displacement vector of the nodes

F load vector of the nodes

a mass ratio of Rayleigh damping

$b \quad$ stiffness ratio of Rayleigh damping

$\zeta_{j} \quad$ damping ratio

$\omega_{j} \quad$ natural frequency

$\Delta t \quad$ fixed integration times step

$T_{\min } \quad$ minimum natural period of all participating modes

$\rho \quad$ fluid density

$D \quad$ riser diameter

$C_{D} \quad$ drag coefficients

$C_{A} \quad$ added mass coefficients

$C_{i} \quad$ lift force coefficients

$V_{m}(z, n)$ modal reduced velocity

$f_{n} \quad$ natural frequency

$r_{h}(z) \quad$ hydrodynamic damping

$r_{s}(z) \quad$ structural damping

$L_{n} \quad$ modal excitation length

$\phi_{n}(z) \quad$ modal shape

\section{References}

Bourguet, R., Karniadakis, G.E., Triantafyllou, M.S., 2013. Multi-frequency vortexinduced vibrations of a long tensioned beam in linear and exponential shear flows. J. Fluid Struct. 41, 33-42.

Chaplin, J.R., Bearman, P.W., Huera-Huarte, F.J., Pattenden, R.J., 2005. Laboratory measurements of vortex-induced vibrations of a vertical tension riser in a stepped current. J. Fluid Struct. 21, 3-24.

Chen, W.M., Li, M., Zheng, Z.Q., 2012. Dynamic characteristics and VIV of deepwater riser with axially varying structural properties. Ocean Eng. 42, 7-12.

Chen, W.M., Li, M., Zhang, L.W., Tan, T.C., 2016. Study on multi-mode VIV of deepwater riser in different flow fields by finite element simulations. J. Offshore Mech. Arctic Eng. 138 (1), 1-8.

Craig Jr., R.R., 1981. Structural Dynamics. Wiley, New York.

Dong, S., Karniadakis, G.E., 2005. DNS of flow past a stationary and oscillating cylinder at $\mathrm{Re}=10000$. J. Fluid Struct. 20, 519-531.

Facchinetti, M., 2004. Vortex-induced traveling waves along a cable. Eur. J. Mech. B Fluid $16,199-208$.

Huang, S., Khorasanchi, M., Herfjord, K., 2011. Drag amplification of long flexible riser models undergoing multi-mode VIV in uniform currents. J. Fluid Struct. 27, 342-353.

Huera-Huarte, J., Bearman, P.W., 2009. Wake structures and vortex-induced vibrations of a long flexible cylinder- Part 2: drag coefficients and vortex modes. J. Fluid Struct. 25 (6), 991-1006.

Huera-Huarte, F.J., Bearman, P.W., Chaplin, J.R., 2006. On the force distribution along the axis of a flexible circular cylinder undergoing multi-mode vortex-induced vibrations. J. Fluid Struct. 22, 897-903.

Huse, E., Kleiven, G., Nielsen, F.G., 1998. Large scale model testing of deep sea risers. In: Proceedings of the Offshore Technology Conference, Houston, Texas, OTC 8701.

Jaiswal, V., Vandiver, J.K., 2007. VIV response prediction for long risers with variable damping. In: Proceedings of the 26th International Conference on Offshore Mechanics and Arctic Engineering OMAE2007-29353, pp. 1-9.

Lie, H., Kaasen, H.K., 2006. Model analysis of measurements from a large-scale VIV model test of a riser in linearly sheared flow. J. Fluid Struct. 22, 557-575.
Lucor, D., Karniadakis, G.E., 2004. Predictability and uncertainty in flow-structure interactions. Eur. J. Mech. B Fluid 23 (1), 41-49.

Lucor, D., Imas, L., Karniadakis, G.E., 2001. Vortex dislocations and force distribution of a long flexible cylinders subjected to sheared flows. J. Fluid. Struct. Mech. 15, 641-650.

Marcollo, H., Hinwood, J.B., 2002. A facility for evaluation of modal competition in an elastic riser. In: Proceedings of the Twelfth (2002) International Offshore and Polar Engineering Conference Kitakyushu, Japan, May, pp. 26-31.

Mukhopadhyay, A., Venugopal, P., Vanka, S.P., 1999. Numerical study of vortex shedding from a circular cylinder in linear shear flow. J. Fluid Eng. Trans. ASME 121, 460-468.

Sarpkaya, T., 2004. A Critical review of the intrinsic nature of vortex-induced vibration. J. Fluid. Struct. Mech. 19, 389-447.

Srinil, N., 2010. Multi-mode interactions in vortex-induced vibrations of flexible curved/ straight structures with geometric nonlinearities. J. Fluid Struct. 26, 1098-1122.

Tang, Y.G., Zhang, S.X., Zhang, R.Y., Liu, H.X., 2007. Development of study on the dynamic characteristics of deep water mooring system. J. Mar. Sci. Appl. 6 (3), 17-23.

Tognarelli, M.A., Slocum, S.T., Frank, W.R., Campbell, R.B., 2004. VIV response of a long flexible cylinder in uniform and linearly sheared currents. In: Offshore Technology Conference. OTC-16338, pp. 1-12.

Trim, A.D., Braaten, H., Lie, H., et al., 2005. Experimental investigation of vortex-induced vibration of long marine risers. J. Fluid Struct. 21, 335-361.

Vandiver, J.K., Li, L., 1999. SHEAR7 Program Theory Manual. MIT, Department of Ocean Engineering.

Vandiver, J.K., Allen, D., Li, L., 1996. The occurrence of lock-in under highly sheared conditions. J. Fluid Struct. 10, 555-561.

Violette, R., de Langre, E., Szydlowski, J., 2010. A linear stability approach to vortexinduced vibrations and waves. J. Fluid Struct. 26 (3), 442-466.

Williamson, C.H.K., Govardhan, R., 2008. A brief review of recent results in vortexinduced vibrations. J. Wind Eng. Ind. Aerod. 96 (5), 713-735.

Zhang, J., Tang, Y.G., Huang, L., Li, W., 2013. Multi-mode coupled vibration behavior of a deep-water riser under parametric excitations. J. Vib. Shock 32 (19), 51-56 (In Chinese). 